\title{
Pin2 telomeric repeat factor 1-interacting telomerase inhibitor 1 (PinX1) inhibits nasopharyngeal cancer cell stemness: implication for cancer progression and therapeutic targeting
}

Chaosheng Yu ${ }^{1+}$, Fang Chen ${ }^{2+}$ (D), Xiaoqi Wang ${ }^{1+}$, Zhimou Cai ${ }^{1}$, Mengxue Yang ${ }^{1}$, Qingwen Zhong ${ }^{1}$, Jialian Feng ${ }^{1}$, Junzheng $\mathrm{Li}^{2^{*}}$, Congxiang Shen ${ }^{1 *}$ and Zhong Wen ${ }^{1 *}$

\begin{abstract}
Background: Recurrence and distant metastasis are still the main factors leading to treatment failure for malignant tumors including nasopharyngeal carcinoma (NPC). Therefore, elucidating the molecular mechanisms underlying nasopharyngeal carcinoma metastasis is of great clinical significance for targeted gene therapy and prognostic evaluation. PinX1, a tumor suppressor gene, was previously demonstrated to be a powerful tool for targeting telomerase in order to resist malignant tumor proliferation and migration. The aim of this study was to explore the mechanism through which PinX1 regulates epithelial-mesenchymal transition (EMT) and tumor metastasis in NPC and investigate its clinical significance and biological role with respect to disease progression.
\end{abstract}

Methods: Cell Counting Kit-8 (CCK8), Transwell assays, Colony formation analysis and Xenograft tumorigenicity assay were used to measure the nasopharyngeal $\mathrm{CD} 133^{+}$cancer stem cell proliferation, migration, and invasion abilities. Reverse transcription-quantitative polymerase chain reaction (RT-qPCR) and western blot assays were conducted to investigate the underlying mechanism that PinX1 inhibits cell proliferation, migration, and invasion via regulating EMT in nasopharyngeal $\mathrm{CD} 133^{+}$CSCS.

Results: We found that the overexpression of PinX1 and P53 inhibited cell proliferation, migration, and invasion, but that the inhibition of miR-200b blocked these effects, in nasopharyngeal CD133 ${ }^{+}$cancer stem cells (CSCs). Mechanistic investigations elucidated that PinX 1 inhibits cell proliferation, migration, and invasion by regulating the P53/miR-200b-mediated transcriptional suppression of Snail1, Twist1, and Zeb1, consequently inhibiting EMT in nasopharyngeal CD133 ${ }^{+}$CSCS.

Conclusions: Our findings indicate that PinX1 inhibits cell proliferation, migration, and invasion via P53/miR-200bregulated EMT in the malignant progression of human NPC, which might suggest novel clinical implications for disease treatment.

Keywords: PinX1, Nasopharyngeal carcinoma, miR-200b, Migration, Invasion, Transcriptional regulation

\footnotetext{
* Correspondence: wenzhong60@163.com; scxiang@126.com; jzli2002@163.com

${ }^{+}$Chaosheng Yu, Fang Chen and Xiaoqi Wang contributed equally to this work.

${ }^{1}$ Department of Otorhinolaryngology-Head and Neck Surgery, Zhujiang Hospital, Southern Medical University, Guangzhou 510282, China

${ }^{2}$ Department of Otorhinolaryngology-Head and Neck Surgery, Guangzhou

Red Cross Hospital, Medical College, Jinan University, Guangzhou 510235,

China
}

(c) The Author(s). 2020 Open Access This article is distributed under the terms of the Creative Commons Attribution 4.0 International License (http://creativecommons.org/licenses/by/4.0/), which permits unrestricted use, distribution, and reproduction in any medium, provided you give appropriate credit to the original author(s) and the source, provide a link to the Creative Commons license, and indicate if changes were made. The Creative Commons Public Domain Dedication waiver (http://creativecommons.org/publicdomain/zero/1.0/) applies to the data made available in this article, unless otherwise stated. 


\section{Background}

Nasopharyngeal carcinoma (NPC) is a malignant tumor of the head and neck, which is derived from epithelial cells located in the nasopharynx, accompanied by early distant metastasis and local invasion, and associated with a high incidence in southern China [1]. Some theoretical evidence demonstrates that the tumorigenesis of NPC is associated with Epstein-Barr virus infection, tumor suppressors, oncogenes, and environmental factors [2, 3]. Combined with chemotherapy technology, a comprehensive treatment plan based on intensity-modulated radiation therapy has achieved excellent local control of nasopharyngeal carcinoma [4]. However, tumor invasion and distant metastasis are still major challenges for successful treatment. Further, the molecular mechanisms underlying tumor invasion and metastasis in NPC need to be completely clarified.

Increasing evidence has indicated that the process of epithelial-mesenchymal transition (EMT), characterized by loss of the epithelial marker E-cadherin and the gain of mesenchymal markers vimentin and $\mathrm{N}$-cadherin, plays an important role in the development of tumor invasion and metastasis for cells of various cancers including NPC [5-7]. Through EMT, epithelial cells lose cell polarity and adhesiveness and thus transform into mesenchymal cells, which might induce a cancer stem cell (CSC) phenotype in tumor cells [8], leading to invasive and metastatic CSCs $[9,10]$. For example, Zhang et al. [11] demonstrated that leucine-rich repeat-containing $G$ protein-coupled receptor 5 (LGR5), a stem cell marker for colon cancer and gastric cancer, can promote EMT by activating the Wnt/beta-catenin pathway in glioma stem cells. These findings suggest a vital link between EMT and the stemness of tumor cells $[11,12]$. EMT and CSCs are key factors in cancer metastasis and invasion; however, the mechanism connecting EMT to stemness remains unclear. Therefore, it is imperative to investigate the molecular mechanisms that drive EMT and tumorinitiating capacity, which might have significant implications for the exploration of new therapeutic targets for the treatment of epithelial malignancies and metastasis.

In our previous work [13], we investigated the role of Pin2/telomeric repeat factor 1-interacting telomerase inhibitor 1 (PinX1) in nasopharyngeal CD $133^{+}$CSCs and found that its overexpression could inhibit proliferation, migration, and invasion and induce apoptosis by significantly downregulating c-Myc expression and upregulating TRF1, Mad1, and P53 expression. However, the underlying mechanisms through which PinX1 regulates EMT and stemness in NPC have not been fully elucidated.

MicroRNA-200b (miR-200b) has recently been found to be highly involved in EMT, tumor metastasis, CSC self-renewal, and differentiation [14-16]. For example, the overexpression of miR-200b was demonstrated to significantly inhibit tumor cell growth and differentiation by targeting GATA-4 to downregulate the expression of CCND1 [16]. In addition, miR-200b has also been found to suppress cell growth, migration, and invasion by targeting Notch1 in NPC [17]. Moreover, increased evidence indicates that the tumor-suppressor P53 can directly regulate miRNAs, which plays crucial roles in tumor initiation, progression, and metastasis [18, 19]. Accordingly, we hypothesized that PinX1 might regulate EMT and tumor metastasis in NPC through the functions of miR-200b and P53.

Currently, few studies have explored the potential mechanisms associated with the cooperation among PinX1, miR-200b, and P53 during the regulation of EMT and tumor metastasis in NPC. Therefore, we investigated the effects of PinX1, miR-200b, and P53 on EMT in nasopharyngeal $\mathrm{CD}_{133^{+}} \mathrm{CSC}$, aiming to provide new therapeutic targets to prevent distant metastasis and NPC progression. In this study, we found that the overexpression of PinX1 and P53 could inhibit nasopharyngeal $\mathrm{CD} 133^{+} \mathrm{CSC}$ proliferation, migration, and invasion but that the inhibition of miR-200b blocked these effects. Furthermore, we showed that PinX1 inhibits cell proliferation, migration, and invasion by regulating the P53/miR-200b-mediated transcriptional suppression of Snail1, Twist11, and Zeb1, ultimately inhibiting EMT to repress the migration and invasion of these cells.

\section{Materials and methods}

\section{Cell culture and transfection}

Nasopharyngeal $\mathrm{CD}_{133^{+}}$cancer stem cells (CSCs) and CD133- CSCs were sorted from the nasopharyngeal cancer cell line CNE2 (poorly differentiated nasopharyngeal squamous cell carcinoma cell line; Beijing Concord Cell Resource Center) using magnetic beads in our previous work [13]. The cells were cultured in DMEM (HyClone, USA) supplemented with $5 \%$ fetal calf serum (Gibco, USA) in a humidified incubator with $5 \% \mathrm{CO}_{2}$ at $37^{\circ} \mathrm{C}$. The culture medium was replaced after $24 \mathrm{~h}$, and cells were passaged after $72 \mathrm{~h}$. Third passages of nasopharyngeal CSCs at the logarithmic phase of growth were divided into the following groups: $\mathrm{CD}_{133^{-}} \mathrm{CSCs}$ (CD133- CSCs without any transfection), blank (CD133 ${ }^{+}$ CSCs without any transfection), negative control (NC, $\mathrm{CD}_{133^{+}} \mathrm{CSCs}$ transfected with empty vector), PinX1 overexpression $\left(\mathrm{CD}_{133^{+}} \mathrm{CSCs}\right.$ transfected with pcDNA3.0-PinX1), P53 overexpression CD133 ${ }^{+}$CSCs transfected with pcDNA3.0-P53), miR-200b inhibitor $\left(\mathrm{CD} 133^{+} \mathrm{CSCs}\right.$ transfected with miR-200b inhibitor), PinX1 overexpression + P53 overexpression $\left(\mathrm{CD} 133^{+}\right.$ CSCs transfected with pcDNA3.0-PinX1 and pcDNA3.0P53) and PinX1 overexpression + miR-200b inhibitor $\left(\mathrm{CD} 133^{+} \mathrm{CSCs}\right.$ transfected with pcDNA3.0-PinX1 and 
miR-200 inhibitor). The plasmids used were synthetized by Shanghai Sangon Biological Engineering Technology \& Services Co., Ltd. (Shanghai, China). All plasmids were transfected into cells according to the instructions of Lipofectamine $^{\text {tw }} 2000$ (Invitrogen, Carlsbad, California, USA). After transfection for $48 \mathrm{~h}$, the cells were harvested and used for subsequent studies. The miR-200b inhibitor (5'-AGAGCUAGCACCAGUAUUA-3') was designed and synthesized by Suzhou GenePharma Co., Ltd. (Suzhou, China).

\section{CCK8 analysis}

The proliferative capacity of nasopharyngeal $\mathrm{CD} 133^{+}$ CSCs and the transfected CSCs was measured by Cell Counting Kit-8 (CCK8, GlpBio, USA) analysis. Briefly, after culture for 24,48 , and $72 \mathrm{~h}$, the cells were seeded on 96-well plates at $1 \times 10^{4}$ cells per well. CCK8 was added to the plates and the plates were incubated for 2 $\mathrm{h}$. The absorbance at $450 \mathrm{~nm}$ was determined using a microplate reader. The experiment was repeated three times to obtain the mean values. The time point was regarded as the abscissa, the OD value as the ordinate, and cell viability curves were plotted.

\section{Migration and invasion assays}

The migration and invasion of nasopharyngeal $\mathrm{CD} 133^{+}$ CSCs and transfected CSCs were determined by Transwell assays. For migration analysis, Six hundred microliters of DMEM supplemented with $10 \%$ fetal bovine serum (FBS) was added to the lower chamber, while $2 \times$ $10^{4}$ cells in serum-free medium were added to the upper chamber. The protocol for the invasion analysis was similar to that of migration analysis, except that the chambers were covered with Matrigel matrix (BD Biosciences, USA). During incubation, the cells migrated and invaded through the lower membrane. Cells on the lower chambers were stained and fixed with $4 \%$ paraformaldehyde and $0.1 \%$ crystal violet, which was followed by counting under an OLYMPUS CX41 upright microscope. A minimum of four fields of vision were randomly selected from each sample to calculate the mean number of cells that had moved through the Matrigel as the index of cell invasion ability.

\section{Colony formation analysis}

Nasopharyngeal $\mathrm{CD}_{133^{+}} \mathrm{CSCs}$ and transfected CSCs were plated in 6 -well plates at $1 \times 10^{4}$ cells per well. The cells were incubated with serum-free DMEM/F12 medium supplemented with HGF $(20 \mathrm{ng} / \mathrm{mL})$, bFGF $(20$ $\mathrm{ng} / \mathrm{mL})$, insulin $(10 \mathrm{ng} / \mathrm{mL})$, and B27 and cultured for 10 days in a $5 \% \mathrm{CO}_{2}$ humidified incubator at $37^{\circ} \mathrm{C}$. After colony formation was observed, the medium was removed. Cells were washed twice with PBS, fixed with $4 \%$ formaldehyde, and stained with 5\% crystal violet. Colonies containing $>50$ cells were used for counting.

\section{Xenograft tumorigenicity assay in nude mice}

Four-week-old female nude mice with body weight of $17 \mathrm{~g}$ were randomly numbered using earrings. A total amount of $1 \times 10^{4}$ logarithmically growing CD $133^{+}$CSCs without any transfection or transfected with pcDNA3.0PinX1 and transfected with pcDNA3.0-PinX1 and miR200 inhibitor in $0.1 \mathrm{ml} 1640$ medium without FBS were subcutaneously injected into the right side of the same nude mice ( $N=5$ each group), respectively, and tumor size was measured once a week in the feeding environment. At 4 weeks after injection, nude mice were sacrificed and tumor grafts were isolated. The size of tumor grafts was measured using the eq. $V=\left(a^{2 *} \mathrm{~b}\right) / 2$, where a is the short side length of the tumor graft and $b$ is long side length of the tumor graft. Differences in volume of tumor graft in $\mathrm{CD} 133^{+} \mathrm{CSCs}$ and $\mathrm{CD} 133^{+}$CSCs transfected with pcDNA3.0-PinX1 and CD133 ${ }^{+}$CSCs transfected with pcDNA3.0-PinX1 and miR-200b inhibitor injected sides were compared. The animals were provided by the Animal Laboratory of the Southern Medical University. In vivo experiments were approved by the Laboratory Animal Committee and conducted in accordance with the National Laboratory Animal Care and Maintenance Guide.

\section{Reverse transcription-quantitative polymerase chain reaction ( $R T-q P C R)$}

Total RNA was extracted from cultured cells with TRIzol reagent (Invitrogen) according to the manufacturer's instructions and was used as a template for reverse transcription reactions into cDNA following the instructions of the Bestar qPCR RT Kit (Applied Biosystems, Grand Island, NY, USA). RT-qPCR was performed using the Agilent Stratagene Mx3000 real-time qPCR Thermocycle Instrument (Agilent Stratagene, CA, USA) with cDNA as the template and glyceraldehyde-3-phosphate dehydrogenase $(G A P D H)$ as the internal reference. The PCR amplifications were performed using DBI Bestar ${ }^{\circ}$ SybrGreen qPCRmasterMix. The reaction conditions were as follows: pre-degeneration at $95^{\circ} \mathrm{C}$ for $2 \mathrm{~min}$, and 40 cycles of denaturation at $94^{\circ} \mathrm{C}$ for $30 \mathrm{~s}$, annealing at $58^{\circ} \mathrm{C}$ for $20 \mathrm{~s}$, and extension at $72^{\circ} \mathrm{C}$ for $20 \mathrm{~s}$, followed by a final extension at $72{ }^{\circ} \mathrm{C}$ for $10 \mathrm{~min}$. The average threshold cycle $(\mathrm{Ct})$ values from three PCR assays were determined and results were calculated based on the $2^{-\triangle \Delta \mathrm{Ct}}$ method and normalized to GAPDH levels. Primers for PinX1, E-cadherin, Vimentin, Snail1, Twist1, $Z e b 1$, and GAPDH were designed and synthesized by Shanghai Sangon Biotechnology Co., Ltd. (Shanghai, China) (Table 1). 
Table 1 The primer sequences for relative mRNA used in this study

\begin{tabular}{|c|c|c|}
\hline ID & Sequence $\left(5^{\prime}-3^{\prime}\right)$ & Product Length(bp) \\
\hline $\operatorname{Pin} X 1 \mathrm{~F}$ & CCAGAGGAGAACGAAACCACG & 128 \\
\hline $\operatorname{Pin} X 1 \mathrm{R}$ & ACCTGCGTCTCAGAAATGTCA & \\
\hline E-cadherin $\mathrm{F}$ & ACAGGGAGGATITGAGCAC & 107 \\
\hline E-cadherin $\mathrm{R}$ & GATCAGCAGAAGTGTCCCTG & \\
\hline Vimentin F & AGTCCACTGAGTACCGGAGAC & 98 \\
\hline Vimentin $\mathrm{R}$ & CATTTCACGCATCTGGCGTTC & \\
\hline Snail1 $\mathrm{F}$ & ACTGCAACAAGGAATACCTCAG & 242 \\
\hline Snail1 R & GCACTGGTACTTCTTGACATCTG & \\
\hline Twist1 F & GAGCAAGATTCAGACCCTCA & 115 \\
\hline Twist1 R & CTCGTGAGCCACATAGCTG & \\
\hline Zeb1 F & CAGCTTGATACCTGTGAATGGG & 106 \\
\hline Zeb1 R & TATCTGTGGTCGTGTGGGACT & \\
\hline GAPDH F & TGTTCGTCATGGGTGTGAAC & 154 \\
\hline GAPDH R & ATGGCATGGACTGTGGTCAT & \\
\hline
\end{tabular}

\section{Western blot analysis}

Total protein was extracted from $1 \times 10^{6}$ cells using the Radio-Immunoprecipitation Assay (RIPA) lysate (Beyotime, Nanjing, China). Next, protein concentration was determined using a BCA Protein Assay Kit (Beyotime). The pre-treated proteins were added to the sampling wells (each well approximately $20 \mu \mathrm{g}$ ) for protein isolation on a $10 \%$ separation gel $(120 \mathrm{~V})$ and $5 \%$ spacer gel $(100 \mathrm{~V})$ for approximately $2 \mathrm{~h}$. The protein samples were then transferred onto polyvinylidene fluoride membranes (Millipore, USA) and blocked with $5 \%$ non-fat milk for $1.5 \mathrm{~h}$. Next, the membranes were washed and incubated with primary antibodies including rabbit polyclonal anti-PinX1 (dilution, 1:1000), rabbit monoclonal anti-Zeb1 (dilution, 1:1000), rabbit monoclonal anti-Snail1 (dilution, 1: 500), rabbit monoclonal anti-E-cadherin (dilution, 1: 3000), rabbit monoclonal anti-Vimentin (dilution, 1: 1500), rabbit polyclonal anti-Twist1 (dilution, 1:2000) and rabbit monoclonal anti-GAPDH (dilution, 1: 10000) at $4{ }^{\circ} \mathrm{C}$ overnight. The membranes were then washed and incubated with the horseradish peroxidase (HRP)-labeled goat anti-rabbit immunoglobulin G (IgG) secondary antibody (dilution, 1:20000, ab6721) at $37^{\circ} \mathrm{C}$ for $4 \mathrm{~h}$. All aforementioned antibodies were purchased from Abcam Inc. (Cambridge, MA, USA). The target signals were visualized using an enhanced chemiluminescence detection kit (ECL, Beyotime). Densitometric analysis of the bands was carried out using the Gel imaging analysis system. Next, the Gel Doc XR imager system (Bio-Rad Laboratories, Inc., Hercules, CA, USA) was used for imaging and Quantity One (Bio-Rad version 4.6.2) was used for quantitative analysis. The gray value ratio of the target protein to the internal reference (GAPDH) was regarded as the relative protein expression. Experiments were repeated three times to obtain the mean values.

\section{Immunohistochemical staining}

The paraffin-embedded tumor tissues prepared from in vivo experiments were sectioned to a thickness of $4 \mu \mathrm{m}$ and mounted on polylysine-coated slides for immunohistochemistry assays to detect protein expression levels of EMT factors. The indirect streptavidinperoxidase method kit (ZSGB-bio, Beijing, China) was used according to the protocol provided by the manufacturer. Briefly, the sections were deparaffinized in xylene and rehydrated in ethanol of gradient concentrations. Antigen retrieval was performed by heating at $100{ }^{\circ} \mathrm{C}$ in $10 \mathrm{mM}$ citrate buffer (Cwbio, Beijing, China) in a pressure cooker for $20 \mathrm{~min}$. The sections were treated with $3 \% \mathrm{H}_{2} \mathrm{O}_{2}$ for $25 \mathrm{~min}$ to quench endogenous peroxidase activity, and with sheep serum for 30 min to block the non-specific binding. Then, the sections were incubated in a humidity chamber with the following antibodies overnight at $4{ }^{\circ} \mathrm{C}$ : anti-E-cadherin (Cat. No. 20874-1AP, 1:100, PTG, USA), anti-Vimentin (Cat. No. 220311-AP, 1:100, PTG, USA). The biotinylated secondary antibody, horseradish peroxidase streptavidin (Cat. No. ab205718, 1:4000, Abcam, USA), and diaminobenzidine (Cat. No. G1211, Servicebio, China) were used successively as the detection reagents. Finally, the sections were counterstained with hematoxylin (Cat. No. G1004, Servicebio, China) for $1 \mathrm{~min}$. Negative controls without primary antibody were used to exclude nonspecific binding.

\section{Statistical analysis}

All data are shown as the mean \pm SEM. Graphpad Prism 6.0 (GraphPad, Inc., USA) was used for statistical analysis. The statistical analysis methods included Student's t-test and Pearson's correlation analysis.

\section{Results \\ PinX1 is downregulated and EMT is promoted in nasopharyngeal $\mathrm{CD} 133^{+} \mathrm{CSCs}$}

The expression of PinX1, E-cadherin, Vimentin, Snail1, Twist1, and Zeb1 in nasopharyngeal CD133 ${ }^{+}$CSCs and CD133- cells was determined by qRT-PCR and western blot analysis. The mRNA levels of PinX1 and E-cadherin were found to be decreased in nasopharyngeal CD133 ${ }^{+}$ CSCs in comparison to those in CD133- cells, but the mRNA levels of Vimentin, Snail1, Twist1, and Zeb1 were elevated (all $p<0.05$; Fig. 1a). When compared to those in $\mathrm{CD}_{133^{-}}$nasopharyngeal CSCs, the $\mathrm{CD} 133^{+} \mathrm{CSCs}$ showed a decrease in the protein levels of PinX1 and E- 


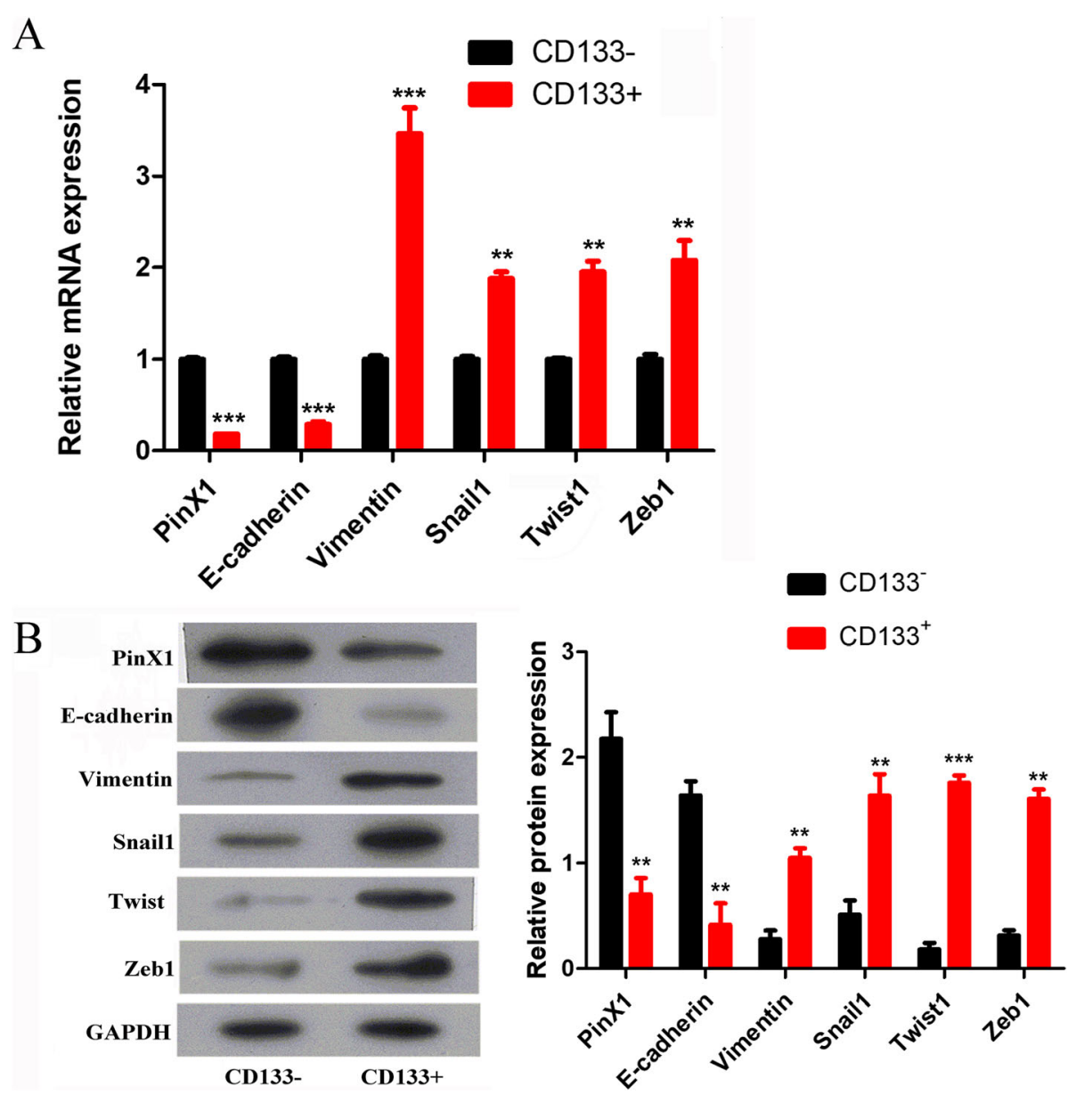

Fig. 1 PinX1 is down-regulated and EMT process is promoted in nasopharyngeal CD133 ${ }^{+}$CSCs. a The mRNA expressions of PinX1, E-cadherin, Vimentin, Snail1, Twist1, and Zeb1 in nasopharyngeal CD133 ${ }^{+}$CSCs and CD133 cells as determined by RT-qPCR. $\mathbf{b}$ The protein levels of PinX1, Ecadherin, Vimentin, Snail1, Twist1, and Zeb1 in nasopharyngeal CD133 $3^{+}$CSCs and CD133- cells as measured by western blot analysis. The grey value of the protein was normalized to that of the corresponding GAPDH. ${ }^{* *} p<0.01$ vs. nasopharyngeal CD133- $\operatorname{CSCs}^{* * *} p<0.001$ vs. nasopharyngeal CD133- CSCs

cadherin and an increase in the protein levels of Vimentin, Snail1, Twist1, and Zeb1 (all $p<0.05$; Fig. 1b). These results proved that PinX1 was poorly expressed while EMT was highly promoted in nasopharyngeal CD133 ${ }^{+}$ CSCs.

\section{Overexpression of PinX1 suppresses EMT by inhibiting EMT-related transcription factors in nasopharyngeal $\mathrm{CD} 133^{+} \mathrm{CSC}$}

We first examined whether PinX1 could regulate EMT in nasopharyngeal $\mathrm{CD} 133^{+} \mathrm{CSCs}$ by transducing them with pcDNA3.0-PinX1. The epithelial marker E-cadherin and the mesenchymal marker Vimentin, related to the EMT process, were detected using RT-qPCR and western blot analyses. E-cadherin was significantly upregulated with PinX1 overexpression, whereas Vimentin showed the opposite pattern, as compared to levels in the blank and empty vector groups (all $p<0.05$; Fig. 2a).
Similar results were found based on western blot assays (all $\mathrm{p}<0.05$; Fig. 2b). These results imply that the overexpression of PinX1 might suppress EMT in nasopharyngeal $\mathrm{CD}_{133^{+}}$CSCs. In our previous work [13], the overexpression of PinX1 was demonstrated to significantly inhibit proliferation, migration, and invasion of these cells, and therefore, PinX1 might inhibit NPC metastasis by suppressing EMT in nasopharyngeal $\mathrm{CD} 133^{+}$ CSCs.

To reveal the potential mechanism through which PinX1 inhibits NPC aggressiveness, the relative mRNA and protein levels of a series of EMT-related transcription factors including Snail1, Twist1, and Zeb1 were further analyzed by RT-qPCR and western blot assays. In nasopharyngeal $\mathrm{CD} 133^{+} \mathrm{CSCs}$ with stable PinX1 overexpression, the EMT-inducing transcription factors Snail1, Twist1, and Zeb1 were significantly downregulated as compared to levels in the blank and empty vector groups 

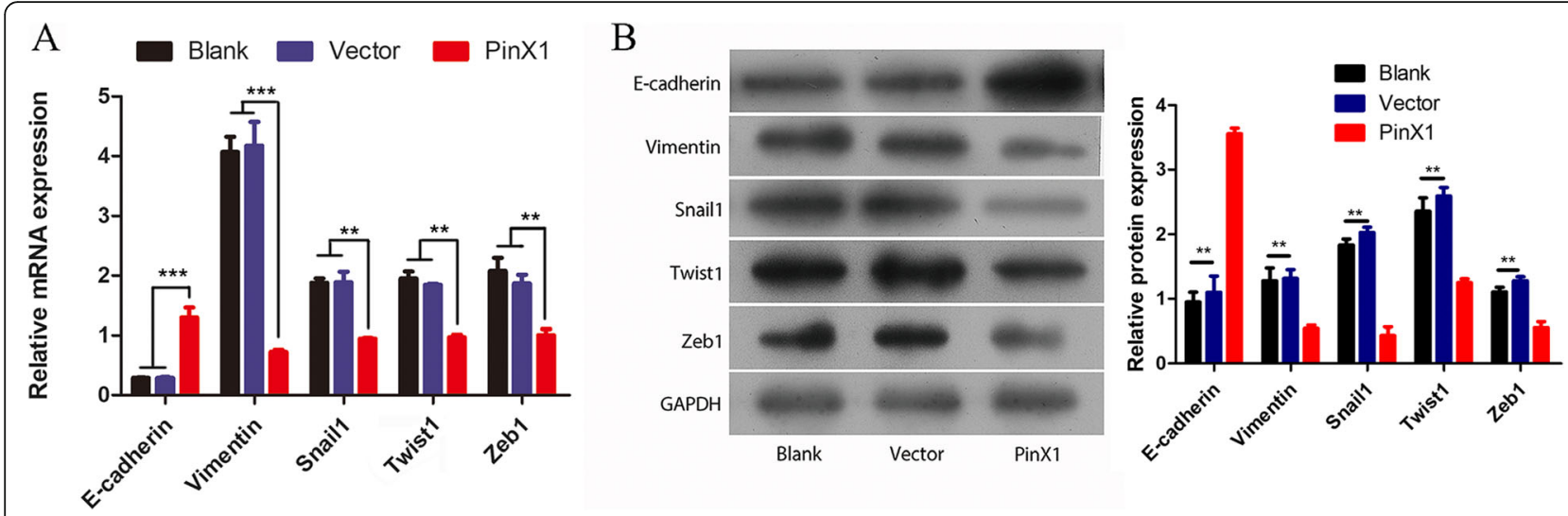

Fig. 2 Overexpression of PinX1 suppresses cell EMT process in nasopharyngeal CD133+ CSCs. a The mRNA expressions of E-cadherin, Vimentin, Snail1, Twist1, and Zeb1 in nasopharyngeal CD133 CSCs and cells transfected with empty vector and pcDNA3.0-PinX1 as determined by RT-qPCR. b The protein levels of E-cadherin, Vimentin, Snail1, Twist1, and Zeb1 in nasopharyngeal CD133 ${ }^{+}$CSCS and cells transfected with empty vector and pcDNA3.0-PinX1 as determined by Western blot. The grey value of the protein was normalized to that of the corresponding GAPDH. ${ }^{* *} \mathrm{p}<$ 0.01 vs. control, ${ }^{* * *} \mathrm{p}<0.001$ vs. control

(all $p<0.05$; Fig. 2a). Similar results were found based on western blot assays (all $\mathrm{p}<0.05$; Fig. 2b). Snail1, Twist1, and Zeb1 can efficiently inhibit the cell-cell adhesion molecule E-cadherin, and this suppression is considered a hallmark of activated EMT. Thus, PinX1 might inhibit Snail1, Twist1, and Zeb1 expression, leading to the deregulation of E-cadherin, and inhibiting nasopharyngeal $\mathrm{CD}_{133^{+}} \mathrm{CSC}$ EMT. These results demonstrated that PinX1 is involved in EMT and that its overexpression can inhibit this process in nasopharyngeal $\mathrm{CD} 133^{+}$ CSCs.

Overexpression of P53 partially promotes the effects of PinX1 overexpression on EMT, migration, and invasion in nasopharyngeal $\mathrm{CD}_{133^{+}} \mathrm{CSCs}$

To further investigate the mechanisms underlying the involvement of PinX1 in nasopharyngeal $\mathrm{CD}_{133^{+}} \mathrm{CSC}$ EMT, we next determined whether P53 expression could affect this process and cell invasion. RT-qPCR and western blot assays showed that the overexpression of P53 downregulated Vimentin expression and upregulated Ecadherin expression similar to that observed with PinX1 overexpression. The expression of EMT-related transcription factors including Snail1, Twist1, and Zeb1 was also significantly repressed with P53 overexpression compared to that in the empty vector group. In addition, pcDNA3.0-PinX1 and pcDNA3.0-P53 co-transfection further inhibited Vimentin, Snail1, Twist1, and Zeb1 expression and promoted E-cadherin expression (Fig. 3a, b). Moreover, the overexpression of P53 significantly inhibited cell migration/invasion ability, and the cooverexpression of P53 and PinX1 further suppressed cell migration/invasion ability (Fig. 3c). Our previous work demonstrated that the overexpression of PinX1 upregulates P53 expression. Thus, PinX1 might upregulate P53 expression in nasopharyngeal CD $133^{+}$CSCs to synergistically inhibit EMT.

The inhibition of miR-200b blocks the effects of PinX1 overexpression on EMT, migration, and invasion in nasopharyngeal $\mathrm{CD} 133^{+} \mathrm{CSCs}$

Further, we investigated whether miR-200b plays an important role in EMT and nasopharyngeal $\mathrm{CD}_{133^{+}} \mathrm{CSC}$ invasion ability. RT-qPCR and western blot analysis showed that the overexpression of PinX1 significantly downregulated Vimentin, Snail1, Twist1, and Zeb1 expression and upregulated E-cadherin expression; however, the co-transfection of pcDNA3.0-PinX1 and a miR-200b inhibitor diminished the effects of PinX1 in nasopharyngeal $\mathrm{CD}_{133^{+}} \mathrm{CSCs}$ (Fig. 4a, b). Moreover, PinX1 overexpression inhibited cell migration/invasion ability; however, pcDNA3.0-PinX1 and miR-200b inhibitor co-transfection prevented these effects in nasopharyngeal $\mathrm{CD} 133^{+} \mathrm{CSCs}$ (Fig. 4c). Thus, these results confirmed that PinX1 suppresses EMT via a miR-200bdependent pathway.

Overexpression of P53 promotes nasopharyngeal CD133 ${ }^{+}$ CSC proliferation and sphere formation ability in vitro but miR-200b inhibition blocks the effects of PinX1

overexpression

Finally, the cell proliferation and in vitro sphere formation ability of nasopharyngeal CD $133^{+} \mathrm{CSCs}$ following transfection were determined via CCK8 and colony formation analyses. The relative viability of nasopharyngeal $\mathrm{CD}_{133^{+}} \mathrm{CSCs}$ following transfection in each group is shown in Fig. 5a. No differences at the 24-h time point were found among the groups $(p>0.05)$. In contrast to that observed in the blank and vector groups, the PinX1, P53, and PinX1 + P53 


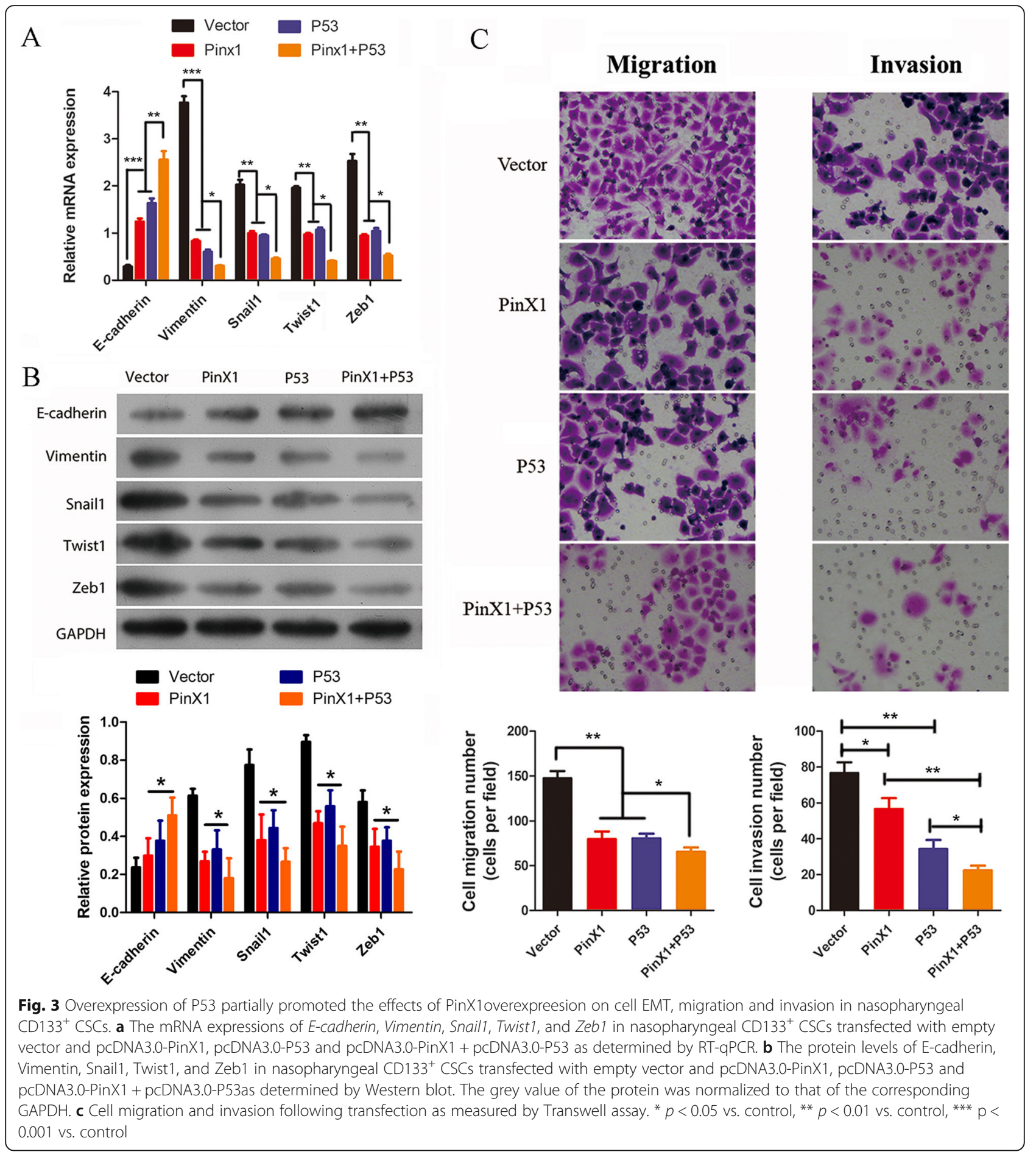

groups exhibited inhibited cell viability at 48 and $72 \mathrm{~h}$ (all $p<0.05$ ). However, this did not differ significantly among the blank, vector, and PinX1 + miR-200b inhibitor groups $(p>0.05)$. To further elucidate the effects of PinX1 on tumor growth, colony formation analyses were performed as shown in Fig. 5b. Compared to those in the blank and vector groups, stem cell spheres of different sizes and irregular shapes were obviously reduced in PinX1, P53, and PinX1 + P53 groups, while no significant differences were observed in the PinX1 + miR-200b inhibitor group. Altogether, the overexpression of PinX1 inhibits nasopharyngeal $\mathrm{CD}_{133^{+}}$cancer stem cell proliferation by activating the miR-200b and P53 pathway. 


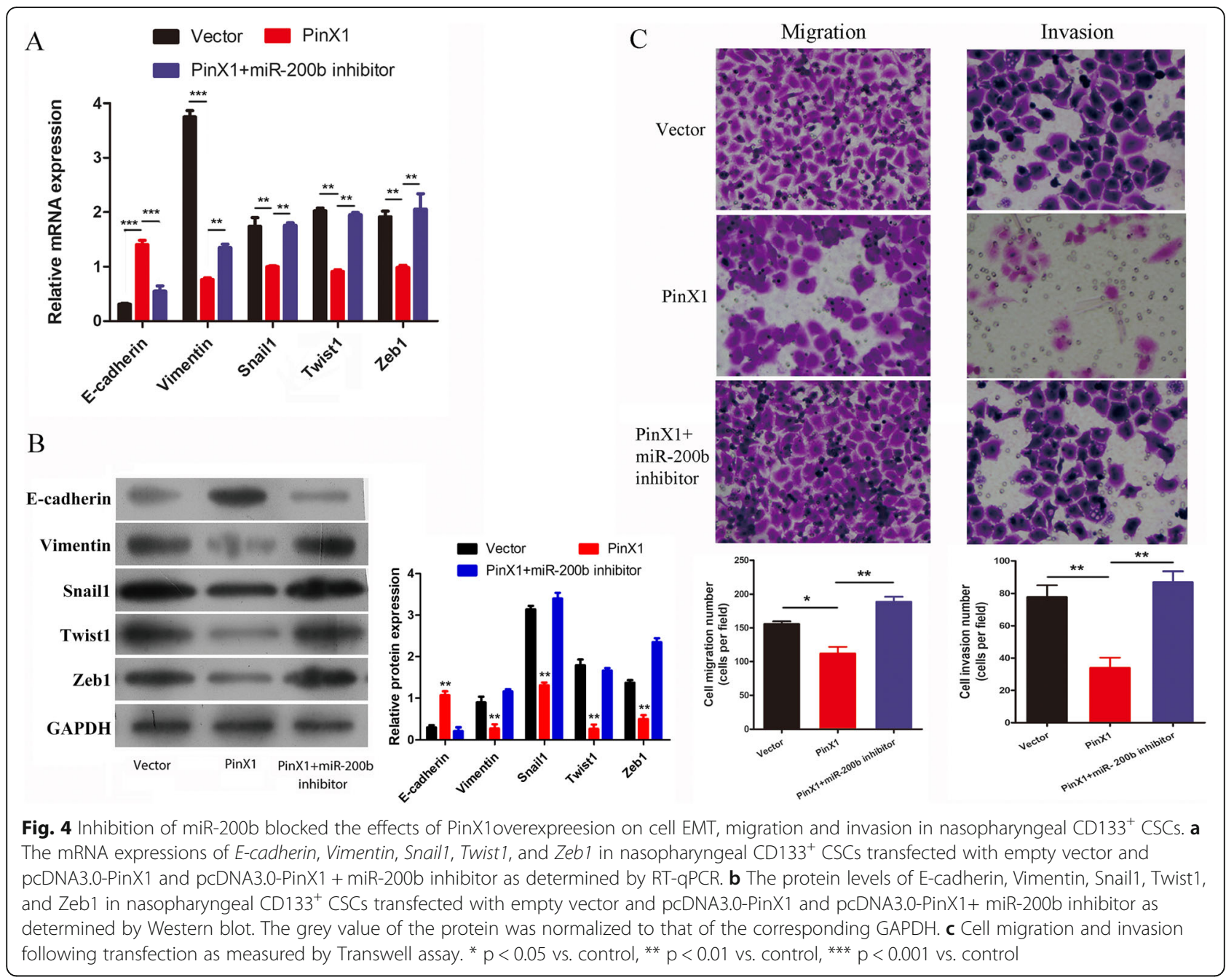

The inhibition of miR-200b blocks the effects of PinX1 overexpression on nasopharyngeal CD $133^{+}$CSCs tumorigenesis and EMT in vivo

Furthermore, an in vivo tumour formation experiment by subcutaneously injecting $\mathrm{CD} 133^{+} \mathrm{CSC}$ without any transfection (Blank group), CD $133^{+}$CSCs transfected with pcDNA3.0-PinX1 (PinX1 group) and CD $133^{+}$CSCs transfected with pcDNA3.0-PinX1 and miR-200b inhibitor (PinX1 + miR-200b inhibitor group) into nude mice were conducted to confirm the effects concerning PinX1 and miR-200b axis (Fig. 6). After 28 days of implantation, the mice injected with PinX1 overexpression cells had smaller tumour burdens than blank group while no significant differences were observed in the PinX1+ miR-200b inhibitor group (Fig. 6a, b). And the growth rate of tumor on mice injected with PinX1 overexpression cells was significantly lower than blank group while no significant differences were observed in the PinX1+ miR-200b inhibitor group (Fig. 6c). In accord with in vitro results, overexpression of PinX1 strongly suppressed the protein and mRNA expression levels of epithelial marker E-cadherin and promoted the expression of mesenchymal marker Vimentin that related to the EMT process in vivo, while the effects could be reversed by co-inhibition of miR-200b (Fig. 6d and e). These tumours also exhibited an increase in E-cadherin expression and a reduction in Vimentin expression in xenografts derived from PinX1 overexpression nasopharyngeal $\mathrm{CD}_{133^{+}} \mathrm{CSCs}$, while the expression levels were reversed by co-inhibition of miR-200b via immunohistochemistry (Fig. 6f). These results showed PinX1 overexpression significantly inhibits tumorigenesis and EMT in vivo while miR-200b inhibition blocks the effects, indicating that miR-200b signal pathway played a vital role on PinX1 regulating aggressive behaviors of nasopharyngeal $\mathrm{CD} 133^{+} \mathrm{CSCs}$.

\section{Discussion}

Although multimodal treatment for the local control of NPC has achieved great advances, with an improved 5year survival rate (approximately 80\%), local recurrence and distant metastasis are still primarily responsible for 


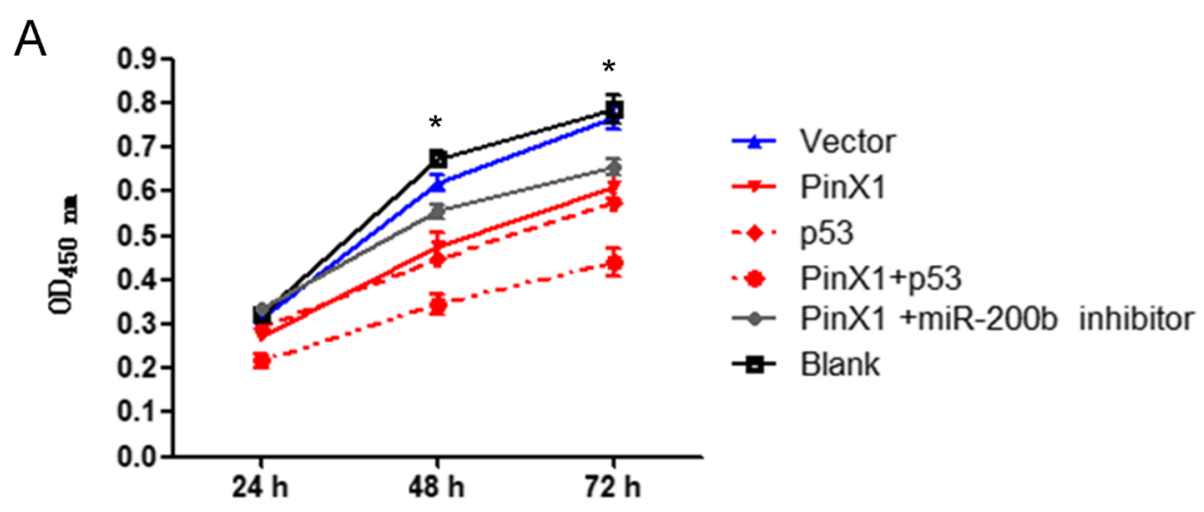

B
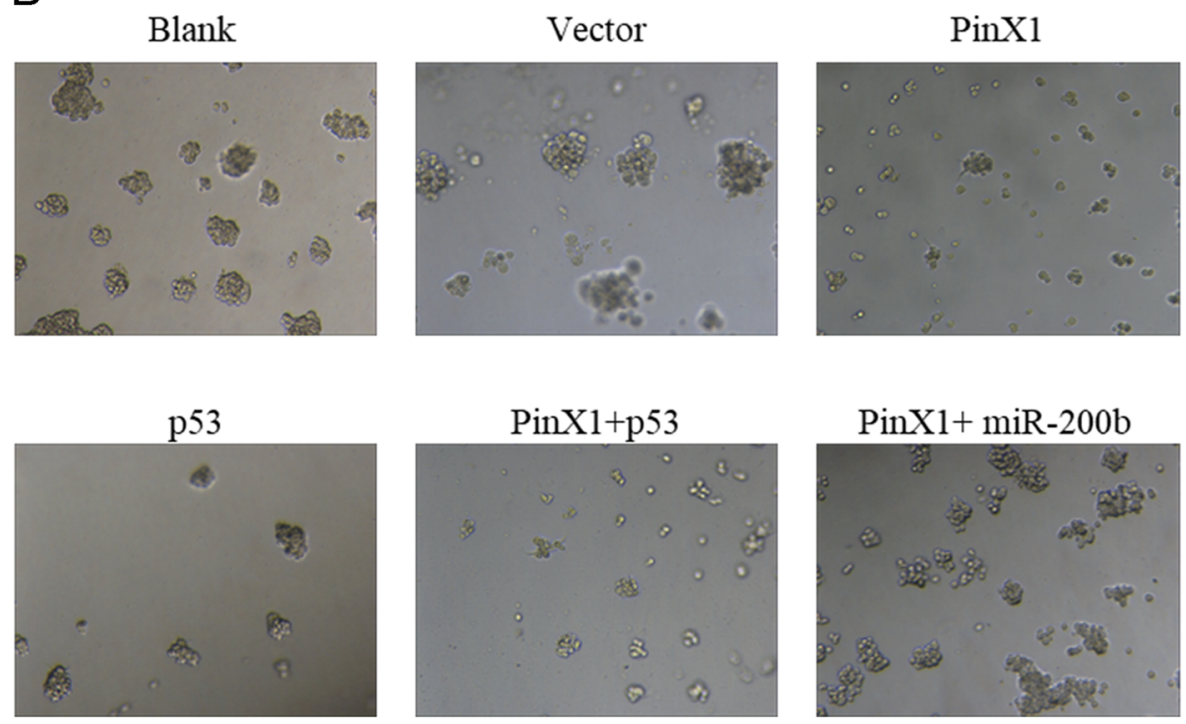

Fig. 5 Overexpression of P53 promoted while inhibition of miR-200b blocked the effects of PinX1 overexpression on cell proliferation and sphere formation ability of nasopharyngeal CD133+ CSCs in vitro. a The cell viability curves of nasopharyngeal CD133+ CSCs following transfection as measured by CCK-8 assay. $\mathbf{b}$ Sphere formation ability of nasopharyngeal CD133 $3^{+}$CSCs following transfection in vitro as determined by colony formation analysis. ${ }^{*} p<0.05$ vs. control

treatment failure and death associated with NPC [20]. Therefore, a better understanding of the underlying mechanism of NPC metastasis is the key to explore novel treatment strategies for patients with this disease. CSCs, a group of "tumor-initiating cells" possessing the capacity to initiate tumor growth, self-renewal properties, and multi-drug resistance, have been found to be highly related to tumor occurrence, development, and metastasis [21]. Over the years, increasing studies have focused on understanding the biological properties and mechanisms of CSC formation to develop novel strategies to identify these stem-like cancer cells and to target them specifically [22-24]. EMT is considered an important process that leads to tumor invasion and distant metastasis, and it has become an important biological characteristic of CSCs that confers new biological behaviors such as chemotherapy resistance, anti- radiation properties, recurrence, and distant metastasis [25]. Guen et al. [26] demonstrated that EMT programs promote basal mammary stem cell and tumor-initiating cell stemness by inducing primary ciliogenesis and Hedgehog signaling. Further, Nomura et al. [27] found that the overexpression of CD133 can increase the expression and secretion of IL1 beta (IL1B), which activates an autocrine signaling loop that upregulates NFkappa B signaling, EMT, and cellular invasion. These results together indicate that EMT and CSCs are mutually interdependent and together confer specific biological behaviors to the tumor.

In this study, we demonstrated that EMT participates in NPC progression. The epithelial marker E-cadherin was decreased, and the mesenchymal marker Vimentin was increased in nasopharyngeal $\mathrm{CD}_{133^{+}} \mathrm{CSCs}$ compared to levels in nasopharyngeal $\mathrm{CD}_{133^{-}}$CSCs. One 


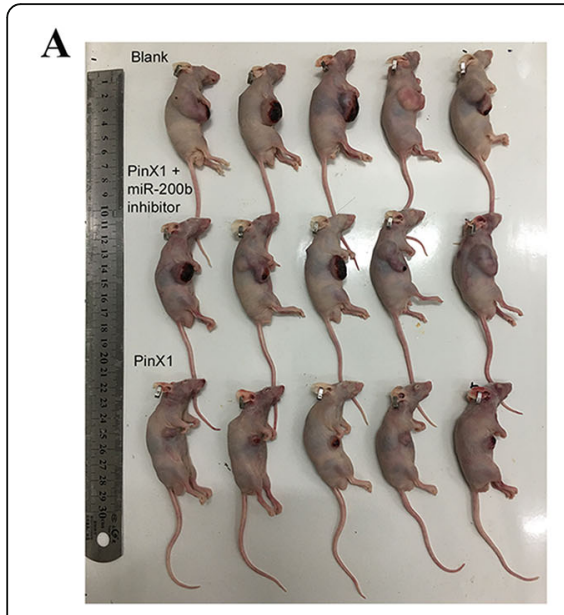

D

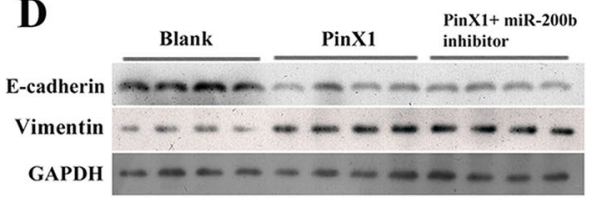

GAPDH
B

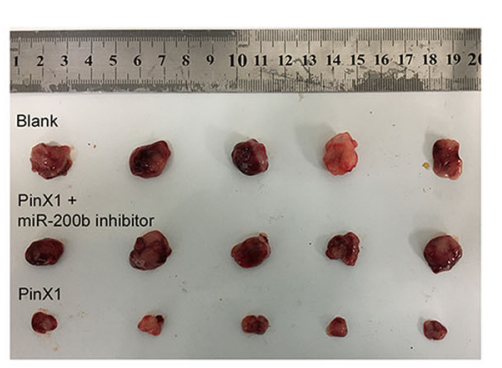

Blank PinX1

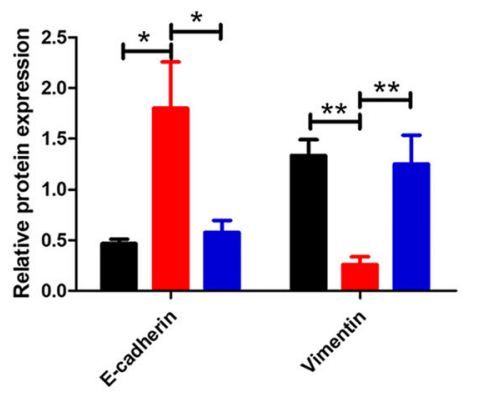

C

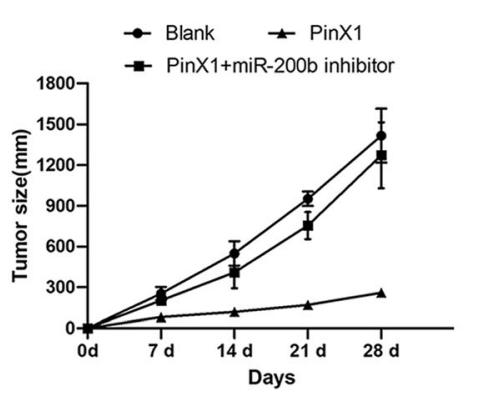

$\mathbf{E}$
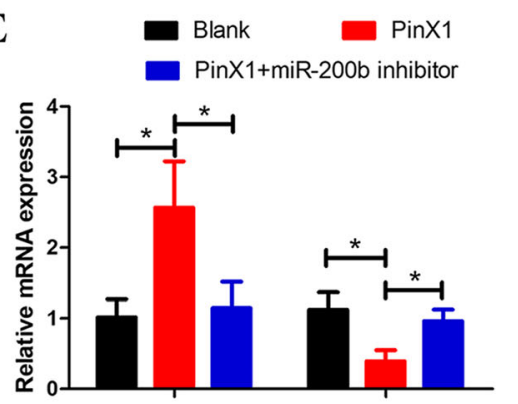

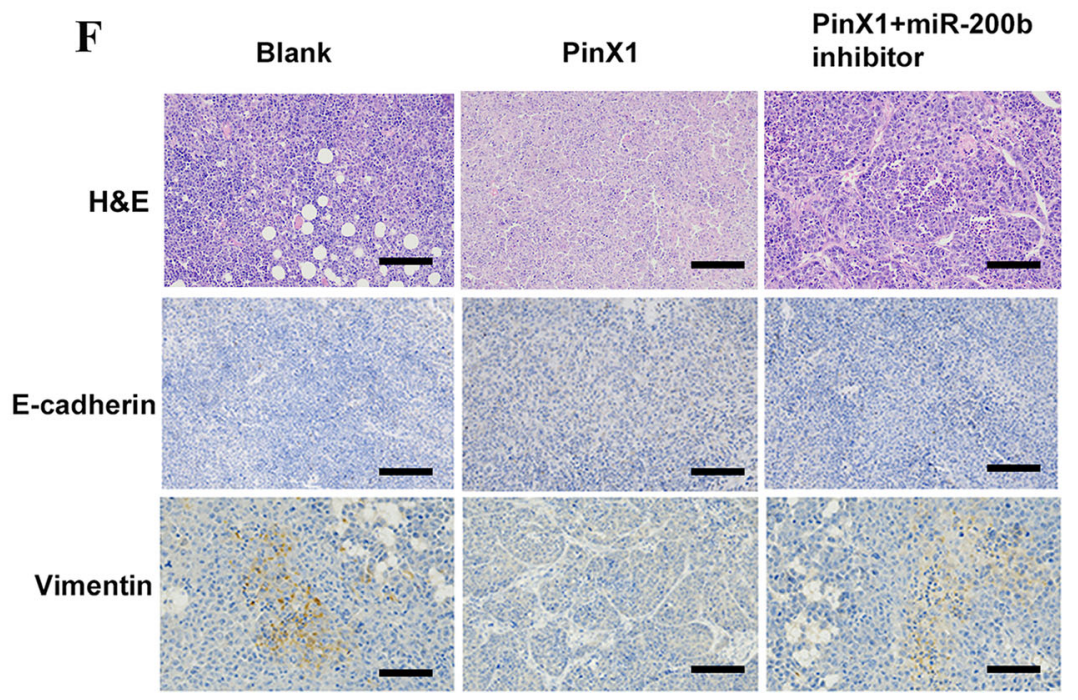

Fig. 6 Inhibition of miR-200b blocked the effects of PinX1 overexpression on cell proliferation and EMT of nasopharyngeal CD133 ${ }^{+}$CSCs in vivo. a The xenograft mice models bearing tumours originating from $\mathrm{CD}_{133^{+}} \mathrm{CSCs}, n=5$ /group. $\mathbf{b}$ tumour volume was periodically measured for each mice. c tumour growth curves was plotted. $\mathbf{d}$ The protein level of Key EMT-related markers was detected by western blot in xenografts derived from nasopharyngeal CD133 ${ }^{+}$CSCs after overexpression of PinX1 and co-inhibition of miR-200b. The grey value of the protein was normalized to that of the corresponding GAPDH. e The mRNA level of Key EMT-related markers was detected by RT-qPCR in xenografts derived from nasopharyngeal $\mathrm{CD} 133^{+}$CSCs after overexpression of PinX1 and co-inhibition of miR-200b. $\mathbf{f}$ Representative H\&E staining of primary cancer tissues are shown as well as immunohistochemistry $(\mathrm{IHC})$ detection of E-cadherin and Vimentin in xenografts derived from nasopharyngeal CD133 CSCs. Magnification $\times 400$. Scale bar: $30 \mu \mathrm{m} .{ }^{*} p<0.05$ vs. control, ${ }^{* *} p<0.01$ vs. control

important characteristic of EMT is the decrease in cadherin expression and the increase in vimentin expression, which indicates that EMT was significantly promoted in nasopharyngeal $\mathrm{CD} 133^{+}$CSCs. To provide a more comprehensive understanding of tumor EMT, a series of EMT-related transcription factors including Snail1, Twist1, and Zeb1 were detected. Twist1 is the most important regulator of EMT and is significantly associated with expression of the mesenchymal markers fibronectin and vimentin [28]. Zhu et al. [29] found that 
Twist11 is involved in EMT in esophageal cancer and cancer-associated fibroblasts and plays a vital role in tumor growth in vivo. Another transcription factor that is closely related to EMT is Snail1, a zinc finger transcriptional repressor that can induce morphological and molecular changes that are characteristic of EMT in breast cancer cells [30]. In addition, Ota et al. [31] found that Snail-induced EMT maintains the CSC-like phenotype, and enhances sphere formation capability, chemoresistance, and invasive ability in head and neck squamous cell carcinoma cells. Moreover, Zeb1, a powerful EMT-related transcription factor, significantly mediates doxorubicin resistance and mesenchymal characteristics in hepatocarcinoma cells [32]. Therefore, understanding the function of transcriptional factors such as Snail1, Twist1, and Zeb1 is necessary to achieve a more comprehensive understanding of tumor EMT. The high expression of Snail1, Twist1, and Zeb1 in nasopharyngeal $\mathrm{CD}_{133^{+}} \mathrm{CSCs}$ indicated that EMT was promoted via the upregulation of these markers.

Telomerase and its core component human telomerase reverse transcriptase (hTERT) are crucial players in cancer metastasis and stemness. El-Badawy et al. [33] investigated the function of hTERT in EMT in CSCs and suggested that targeting this marker might improve the elimination of breast CSCs by coordinating with EMT through a feedback loop. Using gastric cancer as a model, Liu et al. [34] also demonstrated that hTERT stimulates EMT and induces cancer cell stemness, thereby promoting cancer metastasis and recurrence. Therefore, targeting hTERT might prevent cancer progression by inhibiting EMT and CSCs. Our previous work demonstrated that the overexpression of PinX1 significantly decreases hTERT expression, inhibits proliferation, migration, and invasion, and induces apoptosis in nasopharyngeal $\mathrm{CD} 133^{+} \mathrm{CSCs}$ by regulating TRFs and Mad1/c-Myc/P53 pathways [13]. However, the ability of PinX1 to alter the biology of cancer cells needed to be further clarified. Specifically, we found that EMT is significantly inhibited by PinX1 overexpression. Further, in this study, E-cadherin was found to be upregulated and Vimentin, Snail1, Twist1, and Zeb1 were downregulated when PinX1 expression was promoted. Hence, the inhibitory effect on EMT was suggested to be due to PinX1-mediated suppression of the expression of key transcriptional factors involved in this process. In addition, the overexpression of PinX1 was found to diminish the migration and invasion of nasopharyngeal $\mathrm{CD}_{133^{+}}{ }^{\mathrm{CSC}} \mathrm{Cs}$. These results confirmed the role of PinX1 in suppressing tumor aggressiveness in NPC cell lines.

To further reveal the mechanisms underlying the involvement of PinX1 in EMT in nasopharyngeal $\mathrm{CD}_{133^{+}} \mathrm{CSCs}$, the probable related pathways were determined. Since PinX1 was previously found to be associated with P53 and MYC pathways [13], this study further clarified whether P53 is involved in modulating these pathways and suppressing NPC aggressiveness. The expression of E-cadherin was found to be upregulated while the expression of Vimentin and EMT-related transcription factors including Snail1, Twist1, and Zeb1, was significantly suppressed upon P53 overexpression. Co-transfection with pcDNA3.0-PinX1 and pcDNA3.0-P53 further inhibited Vimentin, Snail1, Twist1, and Zeb1 expression and promoted E-cadherin expression. In addition, the overexpression of PinX1 significantly upregulates the expression of $\mathrm{P} 53$ in nasopharyngeal $\mathrm{CD}_{133^{+}} \mathrm{CSCs}$ [13]. These results confirmed that P53 plays a crucial role in PinX1-regulated EMT and tumor aggressiveness in NPC.

Furthermore, this study investigated the function of miR-200b in PinX1-regulated EMT in nasopharyngeal CD133 ${ }^{+}$CSCs. MicroRNAs (miRNAs) are an important class of tumor suppressors or oncogenes that function by regulating their target genes through mRNA degradation, post-transcriptional repression, or promoter activation [35, 36]. These molecules have been recently reported to be closely associated with tumor growth, metastasis, and angiogenesis via transcription factor P53 pathways [37, 38]. Taewan Kim et al. [39] reported that P53 suppresses EMT by transactivating miR-200 family members and then repressing the expression of ZEB1 and ZEB2. Here, we found that the inhibition of miR-200b significantly blocks the effects of PinX1 overexpression on EMT, migration, and invasion in nasopharyngeal $\mathrm{CD} 133^{+}$ CSCs. In addition, the proliferation and in vitro sphere formation abilities of nasopharyngeal $\mathrm{CD} 133^{+}$ CSCs were significantly recovered after inhibiting miR-200b. These data together revealed that miR$200 \mathrm{~b}$ is a key target of PinX1 during the inhibition of EMT.

P53-induced miRNAs played a key role in tumor proliferation, metastasis, and angiogenesis by regulating EMT during the initiation and development of cancer; for example, P53-induced miR-1249 might suppress colorectal cancer (CRC) growth, metastasis, and angiogenesis by targeting VEGFA and HMGA2, in addition to regulating the Akt/mTOR pathway and EMT processes [19]. In addition, Laudato et al. [18] also found that P53-induced miR-30e-5p might inhibit CRC invasion and metastasis by targeting ITGA6 and ITGB1. Here, we observed that the overexpression of PinX1 and P53 inhibits cell proliferation, migration, and invasion, but that the inhibition of miR-200b blocked these effects, in nasopharyngeal CD $133^{+}$CSCs. In addition, we found that PinX1 and P53 inhibit EMT by suppressing Snail1/Twist11/Zeb1 expression. 


\section{Conclusions}

In conclusion, we show here that PinX1 inhibits cell proliferation, migration, and invasion by regulating the $\mathrm{P} 53$ / miR-200b-mediated transcriptional suppression of Snail1, Twist1 and Zeb1, consequently inhibiting EMT, in nasopharyngeal $\mathrm{CD}_{133^{+}}$CSCs. As these processes play a crucial role in the malignant progression of human NPC, our results have showed that P53/miR-200b axis may be a pivotal target for NPC therapy.

\section{Abbreviations}

CCK8: Cell Counting Kit-8; CSCs: cancer stem cells; EMT: Epithelialmesenchymal transition; FBS: Fetal bovine serum; GAPDH: Glyceraldehyde-3phosphate dehydrogenase; hTERT: human telomerase reverse transcriptase; LGR5: Leucine-rich repeat-containing G protein-coupled receptor 5; miR200b: MicroRNA-200b; miRNAs: MicroRNAs; NPC: Nasopharyngeal carcinoma; PinX1: Pin2/telomeric repeat factor 1-interacting telomerase inhibitor 1; RTqPCR: Reverse transcription-quantitative polymerase chain reaction

\section{Acknowledgements}

Not applicable.

\section{Authors' contributions}

CY, FC, XW, JL, CS and ZW developed the conception and design of the study. CY, FC and XW performed most of the experiments (RT-qPCR and Western blot analysis) and analyzed the data. ZC, MY, QZ and JF performed the cell culture and assessment of cell functions in vivo. FC prepared all the figures and wrote the manuscript. JL, CS and ZW reviewed and revised the manuscript. All authors approved the final manuscript.

\section{Funding}

This work was supported by the grants from National Natural Science Foundation of China Youth Fund (81402456) and Science and Natural Science Foundation of Guangdong province, China (2015A030313255) and the Social Development Project of Dongguan (NO. 2016108101025).

\section{Availability of data and materials}

All data generated or analysed during this study are included in this published article.

\section{Ethics approval and consent to participate}

Not applicable.

\section{Consent for publication}

Not applicable.

\section{Competing interests}

The authors declare that they have no competing interests.

Received: 22 August 2019 Accepted: 20 January 2020

Published online: 07 February 2020

\section{References}

1. Wei K-R, Zheng R-S, Zhang S-W, Liang Z-H, Li Z-M, et al. Nasopharyngeal carcinoma incidence and mortality in China, 2013. Chinese J Cancer. 2017; 36:686-93.

2. Hutajulu SH, Indrasari SR, Indrawati LPL, Harijadi A, Duin S, et al. Epigenetic markers for early detection of nasopharyngeal carcinoma in a high risk population. Mol Cancer. 2011;10:48.

3. Coghill AE, Wang C-P, Verkuijilen SAWM, Yu KJ, Hsu W-L, et al. Evaluation of nasal and nasopharyngeal swab collection for the detection of Epstein-Barr virus in nasopharyngeal carcinoma. J Med Virol. 2018;90(1):191-5.

4. Becker-Schiebe $\mathrm{M}$, Christiansen $\mathrm{H}$. Update on combined radio-, Radiochemo- and chemotherapy alone in multimodal therapy of nasopharyngeal carcinoma - a MAC-NPC meta-analysis. Strahlenther Onkol. 2015;191(12):991-3.

5. Liu S-L, Lin H-X, Lin C-Y, Sun X-Q, Ye L-P, et al. TIMELESS confers cisplatin resistance in nasopharyngeal carcinoma by activating the Wnt/beta-catenin signaling pathway and promoting the epithelial mesenchymal transition. Cancer Lett. 2017:402:117-30.

6. Qian W, Ren Z, Lu X. Knockdown of long non-coding RNA TUG1 suppresses nasopharyngeal carcinoma progression by inhibiting epithelialmesenchymal transition (EMT) via the promotion of miR-384. Biochem Biophys Res Commun. 2019;509(1):56-63.

7. Zhou W-M, Wu G-L, Huang J, Li J-G, Hao C, et al. Low expression of PDK1 inhibits renal cell carcinoma cell proliferation, migration, invasion and epithelial mesenchymal transition through inhibition of the PI3K-PDK1-Akt pathway. Cell Signal. 2019:56:1-14.

8. Shuang Z-Y, Wu W-C, Xu J, Lin G, Liu Y-C, et al. Transforming growth factorbeta 1 -induced epithelial-mesenchymal transition generates ALDH-positive cells with stem cell properties in cholangiocarcinoma. Cancer Lett. 2014; 354(2):320-8.

9. Mani SA, Guo W, Liao M-J, Eaton EN, Ayyanan A, et al. The epithelialmesenchymal transition generates cells with properties of stem cells. Cell. 2008;133(4):704-15.

10. Sato R, Semba T, Saya H, Arima Y. Concise review: stem cells and epithelialMesenchymal transition in Cancer: biological implications and therapeutic targets. Stem Cells. 2016;34(8):1997-2007.

11. Zhang J, Cai H, Sun L, Zhan P, Chen M, et al. LGR5, a novel functional glioma stem cell marker, promotes EMT by activating the Wnt/beta-catenin pathway and predicts poor survival of glioma patients. J Exp Clin Cancer Res. 2018;37:225

12. Li Z, Jiang P, Li J, Peng M, Zhao X, et al. Tumor-derived exosomal Inc-Sox2ot promotes EMT and stemness by acting as a ceRNA in pancreatic ductal adenocarcinoma. Oncogene. 2018;37(28):3822-38.

13. Shen C, Chen F, Wang H, Li G, Yu C, et al. The Pinx1 gene Downregulates telomerase and inhibits proliferation of CD133(+) Cancer stem cells isolated from a nasopharyngeal carcinoma cell line by regulating Trfs and Mad1/CMyc/p53 pathways. Cell Physiol Biochem. 2018;49(1):282-94.

14. Feng $X$, Wang $Z$, Fillmore $R, X i$ Y. MiR-200, a new star miRNA in human cancer. Cancer Lett. 2014;344(2):166-73.

15. Chen $J-H$, Zhou L-Y, XU S, Zheng $Y-L$, Wan $Y-F$, et al. Overexpression of IncRNA HOXA11-AS promotes cell epithelial-mesenchymal transition by repressing miR-200b in non-small cell lung cancer. Cancer Cell Int. 2017;17:64.

16. Yao CX, Wei QX, Zhang YY, Wang WP, Xue LX, et al. miR-200b targets GATA-4 during cell growth and differentiation. RNA Biol. 2013;10(4):465-80.

17. Yang $X, N i$ WC, Lei K. miR-200b suppresses cell growth, migration and invasion by targeting Notch1 in nasopharyngeal carcinoma. Cell Physiol Biochem. 2013:32(5):1288-98.

18. Laudato S, Patil N, Abba ML, Leupold JH, Benner A, et al. P53-induced miR$30 \mathrm{e}-5 \mathrm{p}$ inhibits colorectal cancer invasion and metastasis by targeting ITGA6 and ITGB1. Int J Cancer. 2017;141(9):1879-90.

19. Chen X, Zeng K, Xu M, Liu X, Hu X, et al. P53-induced miR-1249 inhibits tumor growth, metastasis, and angiogenesis by targeting VEGFA and HMGA2. Cell Death Dis. 2019;10:131.

20. Lee AWM, Ma BBY, Ng WT, Chan ATC. Management of Nasopharyngeal Carcinoma: current practice and future perspective. J Clin Oncol. 2015; 33(29):3356-64.

21. Yadav AK, Desai NS. Cancer stem cells: acquisition, characteristics, therapeutic implications, targeting strategies and future prospects. Stem Cell Rev. 2019;15(3):331-55

22. Bai $X, N i$ J, Beretov J, Graham P, Li Y. Cancer stem cell in breast cancer therapeutic resistance. Cancer Treat Rev. 2018;69:152-63.

23. Horne GA, Copland M. Approaches for targeting self-renewal pathways in cancer stem cells: implications for hematological treatments. Expert Opin Drug Discovery. 2017;12(5):465-74.

24. Chen K, Huang Y-H, Chen J-L. Understanding and targeting cancer stem cells: therapeutic implications and challenges. Acta Pharmacol Sin. 2013, 34(6):732-40

25. Garg M. Epithelial plasticity and cancer stem cells: major mechanisms of cancer pathogenesis and therapy resistance. World J Stem Cells. 2017;9(8): $118-26$

26. Guen VJ, Chavarria TE, Kroger C, Ye X, Weinberg RA, et al. EMT programs promote basal mammary stem cell and tumor-initiating cell stemness by inducing primary ciliogenesis and hedgehog signaling. Proc Natl Acad Sci U S A. 2017;114(49):E10532-E9.

27. Nomura A, Gupta VK, Dauer P, Sharma NS, Dudeja V, et al. NF kappa Bmediated invasiveness in CD133(+) pancreatic TICs is regulated by 
Autocrine and paracrine activation of IL1 signaling. Mol Cancer Res. 2018; 16(1):162-72.

28. Bulzico D, Barreto Pires BR, Silvestre De Faria PA, Neto LV, Abdelhay E. Twist1 Correlates with epithelial-Mesenchymal transition markers Fibronectin and Vimentin in adrenocortical tumors. Anticancer Res. 2019; 39(1):173-5.

29. Zhu X, Han S, Wu S, Bai Y, Zhang N, et al. Dual role of twist1 in cancerassociated fibroblasts and tumor cells promoted epithelial-mesenchymal transition of esophageal cancer. Exp Cell Res. 2019;375(2):41-50.

30. Mezencev R, Matyunina LV, Jabbari N, McDonald JF. Snail-induced epithelialto-mesenchymal transition of MCF-7 breast cancer cells: systems analysis of molecular changes and their effect on radiation and drug sensitivity. BMC Cancer. 2016;16:236.

31. Ota I, Masui T, Kurihara M, Yook Jl, Mikami S, et al. Snail-induced EMT promotes cancer stem cell-like properties in head and neck cancer cells. Oncol Rep. 2016;35(1):261-6.

32. Long L, Xiang H, Liu JS, Zhang ZM, Sun L. ZEB1 mediates doxorubicin (Dox) resistance and mesenchymal characteristics of hepatocarcinoma cells. Exp Mol Pathol. 2019;106:116-22.

33. El-Badawy A, Ghoneim NI, Nasr MA, Elkhenany H, Ahmed TA, et al. Telomerase reverse transcriptase coordinates with the epithelial-tomesenchymal transition through a feedback loop to define properties of breast cancer stem cells. Biol Open. 2018;7(7):bio034181.

34. Liu Z, Li Q, Li K, Chen L, Li W, et al. Telomerase reverse transcriptase promotes epithelial-mesenchymal transition and stem cell-like traits in cancer cells. Oncogene. 2013;32(36):4203-13.

35. Bartel DP. MicroRNAs: target recognition and regulatory functions. Cell. 2009;136(2):215-33.

36. Peng Y, Croce CM. The role of MicroRNAs in human cancer. Signal Transduction Targeted Ther. 2016;1:15004.

37. He L, He X, Lowe SW, Hannon GJ. microRNAs join the p53 network another piece in the tumour-suppression puzzle. Nat Rev Cancer. 2007;7(11): 819-22.

38. Chang C-J, Chao C-H, Xia W, Yang J-Y, Xiong Y, et al. p53 regulates epithelial-mesenchymal transition and stem cell properties through modulating miRNAs. Nat Cell Biol. 2011;13(3):317-U296

39. Kim T, Veronese A, Pichiorri F, Lee TJ, Jeon YJ, et al. p53 regulates epithelialmesenchymal transition through microRNAs targeting ZEB1 and ZEB2. J Exp Med. 2011;208(5):875-83.

\section{Publisher's Note}

Springer Nature remains neutral with regard to jurisdictional claims in published maps and institutional affiliations.

Ready to submit your research? Choose BMC and benefit from:

- fast, convenient online submission

- thorough peer review by experienced researchers in your field

- rapid publication on acceptance

- support for research data, including large and complex data types

- gold Open Access which fosters wider collaboration and increased citations

- maximum visibility for your research: over $100 \mathrm{M}$ website views per year

At BMC, research is always in progress.

Learn more biomedcentral.com/submissions 